\title{
Forecasting the Long-Run Behavior of the Stock Price of Some Selected Companies in the Malaysian Construction Sector: A Markov Chain Approach
}

\author{
Wajeeh Mustafa Sarsour \\ School of Mathematical Sciences, \\ Universiti Sains Malaysia, Penang, Malaysia. \\ Corresponding author: wajeeh.sa88@gmail.com \\ Shamsul Rijal Muhammad Sabri \\ School of Mathematical Sciences, \\ Universiti Sains Malaysia, Penang, Malaysia. \\ E-mail: rijal@usm.my
}

(Received August 11, 2019; Accepted December 18, 2019)

\begin{abstract}
The fluctuations in stock prices produce a high risk that makes investors uncertain about their investment decisions. The present paper provides a methodology to forecast the long-term behavior of five randomly selected equities operating in the Malaysian construction sector. The method used in this study involves Markov chains as a stochastic analysis, assuming that the price changes have the proparty of Markov dependency with their transition probabilities. We identified a three-state Markov model (i.e., increase, stable, fall) and a two-state Markov model (i.e., increase and fall). The findings suggested that the chains had limiting distributions. The mean return time was computed for respective equities as well as to determine the average duration to return to a stock price increase. The analysis might aid investors in improving their investment knowledge, and they will be able to make better decisions when an equity portfolio possesses higher transition probabilities, higher limiting distribution, and lowest mean return time in response to a price increase. Finally, our investigations suggest that investors are more likely to invest in the GKent based on the three-state model, while VIZIONE seems to be a better investment choice based on a two-state model.
\end{abstract}

Keywords- Stock price, Markov chain, Transition matrix, Expected mean return time.

\section{Introduction}

Recently, investors' interest in the stock market and its performance has arisen. The rising stock market is considered as an indicator of a country's strong economy. Investment and share prices are directly related, that is, a rise in share price will lead to a rise in investments and vice-versa, which makes the stock prices more volatile. Thus, the investment is more likely to be of high risk or likely to suffer from significant losses.

Most of the time, investors have an insight into the behavior of their stocks that will provide guidance in their investment decisions. However, the fluctuations of the stock market are linked to higher investment risks. Therefore, making the correct investment decision significantly depends on an investor's knowledge about the stock market dynamics.

One of the main deriving forces of the Malaysian economy is the construction industry. It plays a significant role in creating wealth and improving the living standards of Malaysians in terms of providing job opportunities for many workers. The Malaysian construction sector exhibited a rapid growth a few years ago as planned according to the Malaysian Vision 2020. Therefore, the 
International Journal of Mathematical, Engineering and Management Sciences

Vol. 5, No. 2, 296-308, 2020

https://doi.org/10.33889/IJMEMS.2020.5.2.024

main objective of the present study is to forecast the long-run behavior of the stock market of several selected companies running in the Malaysian construction sector. The companies selected include PESONA, GKent, SYCAL, VIZIONE, and PSIPTEK. These five respective equities were randomly selected from the Malaysian public listed companies.

The present study seeks to improve investor's knowledge that will help them to make investment decisions with minimum risk that will result in them gaining higher returns. In particular, the decisions of the investors are dependent upon their knowledge of the stock analysis. The analysis of the stocks using statistical models will provide appropriate insight and will increase the investor's knowledge that will sunsequently improve their investment decisions. The statistical models provide information on whether it is wise to invest or not as these models analyse the behavior and trend of the stock prices. One important feature of a competent stock market is that the share prices tend to flactuate randomly, which causes the market information to be uniformly distributed and the forecasting more complex (Bairagi and Kakaty, 2015). A three-state Markov chain model (i.e., price increase, stable, and fall) and a two-state Markov chain model (i.e., increase and fall), which assign the possible transitions of an object from one state to another will take into account its random feature, Markov's dependency, and time-homogeneity. The expected return time of a certain state for each company was computed as well. The data comprised the weekly close prices obtained from the Wall Street Journal's website for all the companies running in the Malaysian construction sector.

Finally, the findings from the present paper might significantly aid investors to improve their investment decisions in the Malaysian construction sector. Given risk minimization, the findings help investors in terms of improving their knowledge and chances of getting higher returns.

\section{Literature Review}

Various methodologies have been developed by several stock market analysts to forecast the long-run behavior of the stock market. Some of the researchers relied on the previous values and behavior of the stocks while others showed that some other factors should be taken into account as well. Recently, there has been a growing debate about the type of methodology that should be used to study the behavior of the stock market. A stochastic stock market predicting model using Markov chains developed by Zhang and Zhang (2009), which forecasts the stock prices in China, is a recognized model that has been used by many researchers. In this model, the transitions system from one state to another of a certain entity is specified using the Markov chain model, which considered the random nature of the transition and stratifies the Markov's dependency property. On the other hand, there are other methods to study the behavior of the stock market such as linear regression and time series models (Akbari, 2013; Sahoo and Charlapally, 2015; Amiri et al., 2016), functional principal component Analysis (Aguilera et al., 1999), and a hidden Markov model (Hassan and Nath, 2005; Xi et al., 2014).

The studies to predict the behavior of the stock prices using Markov Chain models proposed by Zhang and Zhang (2009) are broad. For example, a study by Onwukwe and Samson (2014), a Markov Chain model was applied to investigate the long-run behavior of the closing price of shares for eight banks in Nigeria. They used the initial state vector and the transition probability matrix to compute the limiting distributions for the probability transition matrix of the share price. Additionally, a study by Mettle et al. (2014) had chosen five distinct companies from the Ghana stock exchange market to examine the changes in share prices. The study applied the Markov Chain model with finite states in terms of a stochastic analysis approach for stock price 
International Journal of Mathematical, Engineering and Management Sciences

Vol. 5, No. 2, 296-308, 2020

https://doi.org/10.33889/IJMEMS.2020.5.2.024

change. They showed that all the states found communicate and were ergodic and aperiodic and thus had their limiting distributions. Their results revealed that the use of the Markov Chain model was helpful to improve equity portfolio decisions as well as to further cultivate the investors' knowledge and likelihood of receiving larger returns.

Moreover, Bhusal (2017), explored the long-term behavior of Nepal Stock Exchange (NEPSE) index and found the expected number of visits for each state and the expected time at the first return of different states utilizing daily trading data covering the period between August 15, 2007 to June 18, 2017. The researcher applied the three states Markov Chain model based on the randomness feature of NEPSE index to move from state to another, i.e., increasing or decreasing or remaining the same, by calculating the transition matrix and its $\mathrm{n}^{\text {th }}$ power to evaluate the steady-state for each status. The results were estimated as a probability for each state but they were not regarded as an absolute state. The study conducted by Obasi et al. (2018) had applied a Markov Chain model to investigate the suitability of using this method in investigating and predicting the stock market returns' behavior in the equity market in Nigeria and Ghana. The hypothesis of a random walk of stock prices was tested and a modified Chi-square test was used for both individuals and sector process Markov Chains. They found that the returns of stocks were random and they differed from the indications of the probability vector. Thus, based on these computed probabilities, the model couldn't predict the stock market returns.

\section{Methodology and Data}

\subsection{Markov Chain Model}

A scientist named Andrey Markov developed a theoretical foundation of the Markov chain model in 1906, which was associated with his name. Later in 1936, Kolmogorov had generalized this model to the countable infinite state spaces.

The Markov chain is defined as the sequence $\left\{X_{t}, t=0,1,2, \ldots\right\}$ that is characterised by the property that the future value is conditionally independent of its previous value, given the present value. It can be written mathematically as follows:

$P\left\{X_{t}=j \mid X_{0}=i_{0}, X_{1}=i_{1}, \ldots, X_{t-1}=i_{t-1}\right\}=P\left\{X_{t}=j \mid X_{t-1}=i_{t-1}\right\}$

The Markov chain model is said to have some properties like Markov dependency, which means that the present state depends on the immediate previous state, the transitions are assumed to be stationary in time and do not change over time.

\subsection{Derivation of the MLE for Markov Chains}

Consider that we have a Markov chain model $X_{1}^{\infty}$ for $r$ states. The transition matrix $(p)$ will be calculated from the data with no restrictions. Thus, the number of parameters that we want to estimate are all entries of the matrix $r^{2} ; p_{i j}$. Given the transition probability from one state $i$ to another, $j$ is defined as:

$P\left\{X_{t}=j \mid X_{t-1}=i\right\}=p_{i j}$

Consider the random variable $X_{1}^{n}$ has a realization and a sample $X_{1}^{n} \equiv x_{1}, x_{2}, \ldots, x_{n}$ has been observed from it. This realization would have the probability written as follows:

$$
\begin{aligned}
& P\left[X_{1}^{n}=x_{1}^{n}\right]=P\left[X_{1}=x_{1}\right] \prod_{t=2}^{n} P\left\{X_{t}=x_{t} \mid X_{1}^{t-1}=x_{1}^{t-1}\right\} \\
& =P\left[X_{1}=x_{1}\right] \prod_{t=2}^{n} P\left\{X_{t}=x_{t} \mid X_{t-1}=x_{t-1}\right\}
\end{aligned}
$$


International Journal of Mathematical, Engineering and Management Sciences

Vol. 5, No. 2, 296-308, 2020

https://doi.org/10.33889/IJMEMS.2020.5.2.024

Equation (4) shows the Markov property. Moreover, the maximum likelihood of the transition probability matrix by rewriting Equation (4) in terms of transition probabilities $p_{i j}$,

$L(p)=P\left\{X_{1}=x_{1}\right\} \prod_{t=2}^{n} p_{x_{t-1} x_{t}}$

Consider that the incidence of $i$ is followed by $j$ in $X_{1}^{n}$ denoted by $N_{i j}$. Therefore, the likelihood function will be written as

$L(p)=P\left\{X_{1}=x_{1}\right\} \prod_{i=1}^{r} \prod_{j=1}^{r} p_{i j}^{n_{i j}}$

Taking the logarithm of both sides of Equation (6)

$\log L(p)=\log P\left\{X_{1}=x_{1}\right\}+\sum_{i, j} n_{i j} \log p_{i j}$

Differentiate both sides of Equation (7) with respect to $p_{i j}$, we get

$\frac{\partial \log L(p)}{\partial p_{i j}}=\frac{n_{i j}}{p_{i j}}$

After setting Equation (8) equal to zero, we get

$\frac{n_{i j}}{p_{i j}}=0$

Equation (9) can be solved by eliminating the parameters' framework. Let us select one of the transition probabilities to write in terms of others. Assume it is the probability of going to 1, thus, for every $i, p_{i 1}=1-\sum_{j=2}^{r} p_{i j}$. To differentiate the likelihood function with respect to $p_{i 1}$, we get

$\frac{\partial}{\partial p_{i 1}}=\frac{n_{i j}}{p_{i j}}-\frac{n_{i 1}}{p_{i 1}}$

Now, we can get the maximum likelihood estimator $\hat{p}$ by equating Equation (10) to zero

$\frac{n_{i j}}{\hat{p}_{i j}}=\frac{n_{i 1}}{\hat{p}_{i 1}}$

Hence,

$\frac{n_{i j}}{n_{i 1}}=\frac{\hat{p}_{i j}}{\hat{p}_{i 1}}$

Finally, because this applicable $\forall j \neq 1$, we conclude that $\hat{p}_{i j} \propto n_{i j}$ and thus

$\hat{p}_{i j}=\frac{n_{i j}}{\sum_{j} n_{i j}}$

\subsection{Limiting Distribution}

If we multiply the transition probability matrix $p$ by itself $m$ times, then the resulted matrix is called the m-step transition probability matrix, in which its entries $p_{i j}^{m}$ is the conditional probability that the company will stay in state $j$ after $m$ transitions, given that it is currently in state $i$.

$p_{i j}^{m}=P\left\{X_{t+m}=j \mid X_{t}=i\right\}$

When the class of Markov chain is characterized by aperiodic, irreducible, finite state (i.e., the limit exists), the limiting distribution of such a Markov chain is defined as: 
International Journal of Mathematical, Engineering and Management Sciences

Vol. 5, No. 2, 296-308, 2020

https://doi.org/10.33889/IJMEMS.2020.5.2.024

$\lim _{m \rightarrow \infty} p^{m}=\pi=\left[\begin{array}{c}\boldsymbol{\alpha} \\ \boldsymbol{\alpha} \\ \vdots \\ \boldsymbol{\alpha}\end{array}\right]$

where $\boldsymbol{\alpha}=\left[\begin{array}{llll}\alpha_{1} & \alpha_{2} & \cdots & \alpha_{r}\end{array}\right]$ in which $0<\alpha_{j}<1$ and $\sum_{j=1}^{r} \boldsymbol{\alpha}_{j}=1$; and $r$ is the number of states (Bhat, 1984). Equation (15) is then a chain having an ergodic property and $\pi$ is its limiting distribution.

\subsection{Expected Return Time}

Consider a finite irreducible Markov chain, $\mathrm{p}^{\mathrm{m}}$, and $\pi$ is its limiting distribution, as mentioned in the previous section. After taking the reciprocal of the limiting distribution, we can obtain the mean return time to state $j$ it was firstly in at time 0 (Bhusal, 2017).

$\mu=1 / \pi=\left[\begin{array}{c}1 / \alpha \\ 1 / \alpha \\ \vdots \\ 1 / \alpha\end{array}\right]$

where $\mathbf{1} / \boldsymbol{\alpha}=\left[\begin{array}{llll}1 / \alpha_{1} & 1 / \alpha_{2} & \cdots & 1 / \alpha_{r}\end{array}\right]$ and $r$ is the number of states.

\subsection{Data}

The data comprise of weekly trade closing prices and were obtained from Wall Street Journal website for five randomly selected companies running in the Malaysian construction sector and public listed in Bursa Malaysia covering the period from 1 January 2010 to 31 December 2017. The changes in closing prices were obtained based on the following equation

$\Delta Q_{t}=X_{t}-X_{t-1}$

where $\Delta Q_{t}$ is the close price change, $X_{t}$ is the closing price in week $t$, and $X_{t-1}$ is the closing price in the immediate previous week $t-1$.

The selected companies are PESONA, GKent, SYCAL, VIZIONE, and PSIPTEK. The total number of trading weeks is 418 weeks. The summary statistics of each state (i.e., increase, stable, fall) are presented in Table 1, which includes the minimum, maximum, mean, standard deviations, kurtosis, and skewness for each selected company as well as for the remaining companies running construction sector to explore to the extent to which the stock market is volatile. In general, the stock price movements are more likely to experience decreasing state during the study period. The average weekly price changes and their respective standard deviations for each company listed in Bursa Malaysia are plotted in Figure 1. If the average price changes are close to its standard deviation, then the risk associated with an investment decision is low. Otherwise, it is associated with high risk. It seems that the stock market prices (measured weekly) are highly volatile, which means that investment decisions in such equities are associated with high risks. 
International Journal of Mathematical, Engineering and Management Sciences

Vol. 5, No. 2, 296-308, 2020

https://doi.org/10.33889/IJMEMS.2020.5.2.024

Table 1 . Summary statistics on the weekly trading price change over the study period

\begin{tabular}{|c|c|c|c|c|c|c|c|c|c|c|}
\hline & \multicolumn{3}{|c|}{ Number of weekly price change } & \multicolumn{7}{|c|}{ Weekly price change summary(Malaysian Ringgit-MYR) } \\
\hline & increase & Stable & decrease & Mean & SD & $\operatorname{Max}$ & Min & Skew. & Kurt. & Count. \\
\hline CRESBLD & 158 & 55 & 205 & 0.95 & 0.32 & 1.62 & 0.47 & 0.34 & $\begin{array}{l}-0.88 \\
\end{array}$ & 418 \\
\hline DKLS & 145 & 165 & 108 & 1.43 & 0.32 & 2.12 & 0.95 & 0.05 & -1.67 & 418 \\
\hline EKOVEST & 180 & 39 & 199 & 0.53 & 0.27 & 1.45 & 0.23 & 1.63 & 1.86 & 418 \\
\hline GADANG & 183 & 29 & 206 & 0.57 & 0.34 & 1.31 & 0.22 & 0.82 & -0.64 & 418 \\
\hline FAJAR & 173 & 52 & 193 & 0.73 & 0.22 & 1.22 & 0.35 & 0.29 & -1.18 & 418 \\
\hline PESONA & 160 & 84 & 174 & 0.37 & 0.25 & 1.00 & 0.04 & 0.40 & -0.80 & 418 \\
\hline SYCAL & 146 & 90 & 182 & 0.28 & 0.10 & 0.52 & 0.11 & -0.01 & -1.23 & 418 \\
\hline ZECON & 166 & 54 & 198 & 0.62 & 0.15 & 1.08 & 0.37 & 0.68 & -0.18 & 418 \\
\hline BPHB & 165 & 58 & 195 & 0.73 & 0.30 & 1.54 & 0.34 & 0.62 & -0.62 & 418 \\
\hline GAMUDA & 196 & 26 & 196 & 4.29 & 0.74 & 5.50 & 2.70 & -0.41 & -1.04 & 418 \\
\hline GKent & 202 & 41 & 175 & 0.90 & 0.80 & 3.64 & 0.33 & 1.91 & 2.48 & 418 \\
\hline JETSON & 147 & 78 & 193 & 0.54 & 0.22 & 1.25 & 0.21 & 0.47 & -0.21 & 418 \\
\hline MERCURY & 176 & 73 & 169 & 1.17 & 0.36 & 2.49 & 0.53 & 0.35 & 1.06 & 418 \\
\hline MERGE & 151 & 90 & 177 & 0.35 & 0.12 & 0.67 & 0.15 & 0.92 & 1.42 & 418 \\
\hline HOHUP & 169 & 39 & 210 & 0.89 & 0.33 & 1.87 & 0.42 & 0.82 & -0.18 & 418 \\
\hline HSL & 168 & 57 & 193 & 1.68 & 0.20 & 2.10 & 1.08 & -0.34 & -0.49 & 418 \\
\hline IJM & 194 & 26 & 198 & 3.04 & 0.37 & 3.70 & 2.16 & -0.36 & -1.07 & 418 \\
\hline IREKA & 160 & 64 & 194 & 0.62 & 0.09 & 0.89 & 0.48 & 0.88 & 0.82 & 418 \\
\hline JAKS & 172 & 53 & 193 & 0.77 & 0.33 & 1.66 & 0.33 & 0.90 & -0.07 & 418 \\
\hline KERJAYA & 176 & 84 & 158 & 1.28 & 0.95 & 4.08 & 0.11 & 1.34 & 1.07 & 418 \\
\hline MELATI & 144 & 95 & 179 & 0.87 & 0.21 & 1.48 & 0.55 & 0.80 & 0.19 & 418 \\
\hline MGB & 121 & 186 & 111 & 0.46 & 0.40 & 2.00 & 0.02 & 1.46 & 1.84 & 418 \\
\hline MITRA & 129 & 174 & 115 & 0.75 & 0.47 & 2.01 & 0.17 & 0.56 & -1.02 & 418 \\
\hline MTDACPI & 164 & 64 & 190 & 0.39 & 0.10 & 0.61 & 0.20 & 0.11 & -1.08 & 418 \\
\hline MUDAJYA & 165 & 34 & 219 & 2.23 & 0.94 & 4.41 & 0.77 & 0.09 & -1.20 & 418 \\
\hline MUHIBAH & 191 & 26 & 201 & 1.90 & 0.72 & 3.36 & 0.81 & -0.04 & -1.36 & 418 \\
\hline OCR & 171 & 66 & 181 & 0.35 & 0.15 & 0.72 & 0.13 & 0.44 & -1.34 & 418 \\
\hline PTARAS & 202 & 41 & 175 & 2.59 & 1.28 & 4.60 & 0.68 & -0.11 & -1.65 & 418 \\
\hline PSIPTEK & 158 & 84 & 176 & 0.18 & 0.05 & 0.30 & 0.10 & 0.54 & -0.52 & 418 \\
\hline PRTASCO & 181 & 61 & 176 & 1.05 & 0.25 & 1.67 & 0.70 & 0.33 & -1.06 & 418 \\
\hline PUNCAK & 168 & 33 & 217 & 1.98 & 0.87 & 3.71 & 0.62 & 0.14 & -1.48 & 418 \\
\hline TRC & 165 & 60 & 193 & 0.54 & 0.12 & 0.84 & 0.29 & 0.23 & -0.62 & 418 \\
\hline TSRCAP & 149 & 86 & 183 & 0.74 & 0.16 & 1.25 & 0.45 & 0.14 & -0.07 & 418 \\
\hline VIZIONE & 167 & 91 & 160 & 0.13 & 0.03 & 0.30 & 0.08 & 1.27 & 2.71 & 418 \\
\hline WCEHB & 170 & 50 & 198 & 1.03 & 0.22 & 1.64 & 0.31 & -0.59 & 1.91 & 418 \\
\hline WCT & 188 & 43 & 187 & 1.90 & 0.29 & 2.47 & 1.15 & -0.15 & -0.64 & 418 \\
\hline ZELAN & 154 & 65 & 199 & 0.29 & 0.12 & 0.67 & 0.10 & 0.81 & 0.21 & 418 \\
\hline
\end{tabular}

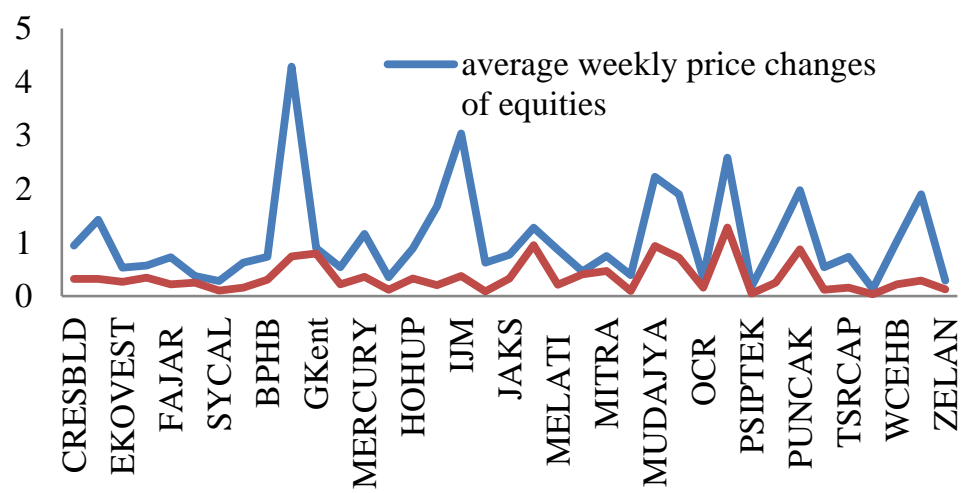

Figure 1. A plot of the mean and standard deviation of weekly price changes of equities 
International Journal of Mathematical, Engineering and Management Sciences

Vol. 5, No. 2, 296-308, 2020

https://doi.org/10.33889/IJMEMS.2020.5.2.024

\subsection{Model Specifications}

\subsubsection{Two States Model}

We can define a two-state Markov model by considering the closing prices, which exhibits either an increase or a fall. The chain is defined as an increase (R) if the closing price of $t^{t h}$ week is higher than or equal to the previous week. In other words, from Equation (17), if the closing price change is higher than or equal to zero it is defined as an increase. Otherwise, it is defined as a fall $(\mathrm{F})$. The pattern of share price movement from fall $(\mathrm{F})$ to rise $(\mathrm{R})$ is labeled by (FR). Based on Equation (17), we can define a discrete random variable, $Z_{t}$, that takes two different values defined as follows:

$Z_{t}= \begin{cases}1, & \text { if } \Delta Q_{t} \geq 0 \\ 2, & \text { if } \Delta Q_{t}<0\end{cases}$

Therefore, the random variable $Z_{t}$ has a Markov chain of two states: 1 and 2. The number of transition from state $i$ to state $j$ is denoted by $n_{i j}$. The structure of the transition matrix is represented by the following equation,

$T=\left[\begin{array}{ll}n_{11} & n_{12} \\ n_{21} & n_{22}\end{array}\right]$

Now, we can obtain the transition probability matrix based on Equation (13), which can be represented as follows,

$p=\left[\begin{array}{ll}p_{11} & p_{12} \\ p_{21} & p_{22}\end{array}\right]$

\subsubsection{Three States Model}

The possible movements of the price change can either be an increase, stable, or fall. These possible movements are considered as the three states of the probability transition matrix of the Markov chain process. The chain is defined as an increase (R), if the closing price of $t^{\text {th }}$ week is higher than the previous week, (i.e., from Equation 17, if the closing price change is higher than zero). If the closing price change is equal to zero, then it is defined as a stable (S). If the closing price change is less than zero, it is defined as a fall (F). The pattern of the movement of share price fall state $(\mathrm{F})$ to increase state $(\mathrm{R})$ is labeled by $(\mathrm{FR})$ and so on.

Based on Equation (17), we can define a discrete random variable, $Z_{t}$, that takes three different values defined as follows:

$Z_{t}= \begin{cases}1, & \text { if } \Delta Q_{t}>0 \\ 2, & \text { if } \Delta Q_{t}=0 \\ 3, & \text { if } \Delta Q_{t}<0\end{cases}$

Therefore, the random variable $Z_{t}$ has a Markov chain of three states 1 (increase), 2 (stable), and 3 (fall). The number of transitions from state $i$ to state $j$ is denoted by $n_{i j}$, and we get the transition matrix for a three-state Markov model.

$T^{*}=\left[\begin{array}{lll}n_{11} & n_{12} & n_{13} \\ n_{21} & n_{22} & n_{23} \\ n_{31} & n_{32} & n_{33}\end{array}\right]$

Now we can obtain the transition probability matrix based on Equation (13), which can be represented as follows, 
International Journal of Mathematical, Engineering and Management Sciences

Vol. 5, No. 2, 296-308, 2020

https://doi.org/10.33889/IJMEMS.2020.5.2.024

$p=\left[\begin{array}{lll}p_{11} & p_{12} & p_{13} \\ p_{21} & p_{22} & p_{23} \\ p_{31} & p_{32} & p_{33}\end{array}\right]$

\section{Results and Discussion}

\subsection{Three-state Transition Matrix (Long-term behaviour)}

The transition probabilities for each one of the five randomly selected companies are shown below.

$$
\begin{aligned}
P_{\text {PENOSA }} & =\left[\begin{array}{ccc}
0.411392 & 0.170886 & 0.417722 \\
0.369048 & 0.27381 & 0.357143 \\
0.356322 & 0.195402 & 0.448276
\end{array}\right], \\
P_{\text {Gkent }} & =\left[\begin{array}{lll}
0.435000 & 0.115000 & 0.450000 \\
0.512195 & 0.195122 & 0.292683 \\
0.525714 & 0.057143 & 0.417143
\end{array}\right], \\
P_{\text {SYCAL }} & =\left[\begin{array}{ccc}
0.3125 & 0.152778 & 0.534722 \\
0.355556 & 0.277778 & 0.366667 \\
0.368132 & 0.236264 & 0.395604
\end{array}\right], \\
P_{\text {VIZIONE }} & =\left[\begin{array}{lll}
0.339394 & 0.157576 & 0.50303 \\
0.417582 & 0.296703 & 0.285714 \\
0.44375 & 0.2375 & 0.31875
\end{array}\right], \\
P_{\text {PSIPTEK }} & =\left[\begin{array}{lll}
0.275641 & 0.134615 & 0.589744 \\
0.416667 & 0.27381 & 0.309524 \\
0.443182 & 0.227273 & 0.329545
\end{array}\right] .
\end{aligned}
$$

It is clear that the chains of all the selected companies are irreducible since their respective estimated transition probability matrices for all the states are greater than zero; i.e., $\hat{p}_{i j}>0$, $\forall i, j=0,1,2$.

For the case of share price increase, the m-step transition probabilities were calculated for the selected companies, relying on time-homogeneity assumption and presented in Figure 2 as a linear plot of transition probabilities for $p_{11}$. The rational choice is to select the company with the highest value of $p_{11}$.

It appears from Figure 2 that GKent company has the highest probability of moving from one high price to another high price, which seems to be the better choice for an investor during the study period. However, the lowest transition probability is recorded by the SYCAL company. Comparing PESONA to PSIPTEK, Figure 2 shows that both shares have almost the same probability of transition despite PSIPTEK shares started at the lowest probability of transition to higher prices during the study period. 
International Journal of Mathematical, Engineering and Management Sciences

Vol. 5, No. 2, 296-308, 2020

https://doi.org/10.33889/IJMEMS.2020.5.2.024

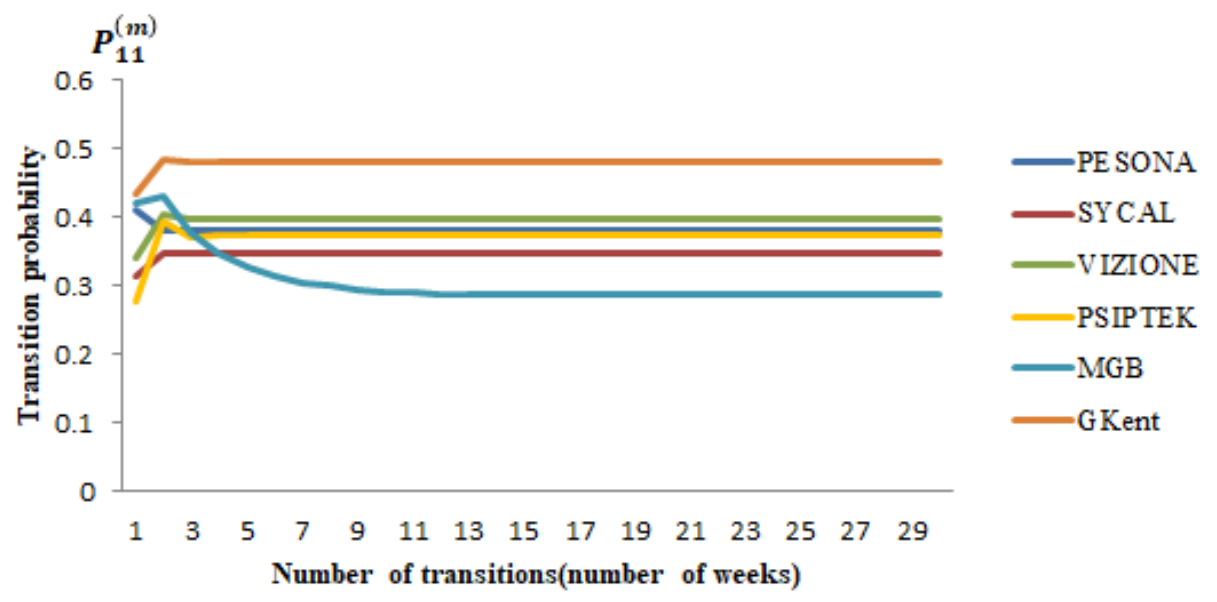

Figure 2. The m-step transition probabilities for share price increases

The percentages of times the company stayed in a specific state in the long-term are exhibited by the limiting distributions of each selected company. As shown in Table 2, GKent company has a $48 \%$ chance of experiencing an increase, and $9.9 \%$ chances of being stable in the long-term. On the other hand, it has a $42.1 \%$ chance of falling in the long-term. Similarly, the PSIPTEK has $37.5 \%, 20.2 \%$, and $42.3 \%$ of experiencing increasing, stable, and falling, respectively in the longterm. The remaining companies were also interpreted analogously. In this regard, it is easily seen that GKent company has the highest probability of increasing the stock prices in the long-term, in comparison to the selected companies in the present study.

Table 2. Entries of the limiting distribution at for respective equities for three-state

\begin{tabular}{|c|ccc|}
\hline Equity & $\alpha 1$ & $\alpha 2$ & $\alpha 3$ \\
\hline PESONA & 0.37981 & 0.20192 & 0.41827 \\
GKent & 0.48077 & 0.09856 & 0.42067 \\
SYCAL & 0.34615 & 0.21635 & 0.43750 \\
VIZIONE & 0.39664 & 0.21875 & 0.38461 \\
PSIPTEK & 0.37500 & 0.20192 & 0.42308 \\
\hline
\end{tabular}

The expected return time $\left(\mu_{i j}\right)$ for each state were also computed, which are shown in Table 3. $\mu_{i j}$ is measured in weeks. Figure 3 illustrates the expected return time for respective shares in the case of a three-state Markov model at $\mu_{11}$, which determines the mean return time until the subsequent increase in the share price occurs. From Figure 3, we can see that the GKent company possesses the lowest mean return time to a price increase. On average, the chain of GKent equity has to visit the increasing state in two weeks. The rational choice of an investor is to choose equity that has the highest transition and limiting distributions, but also that the shortest mean duration to a price increase. Having the minimum mean return time avails the lowest return time to a price increase. 


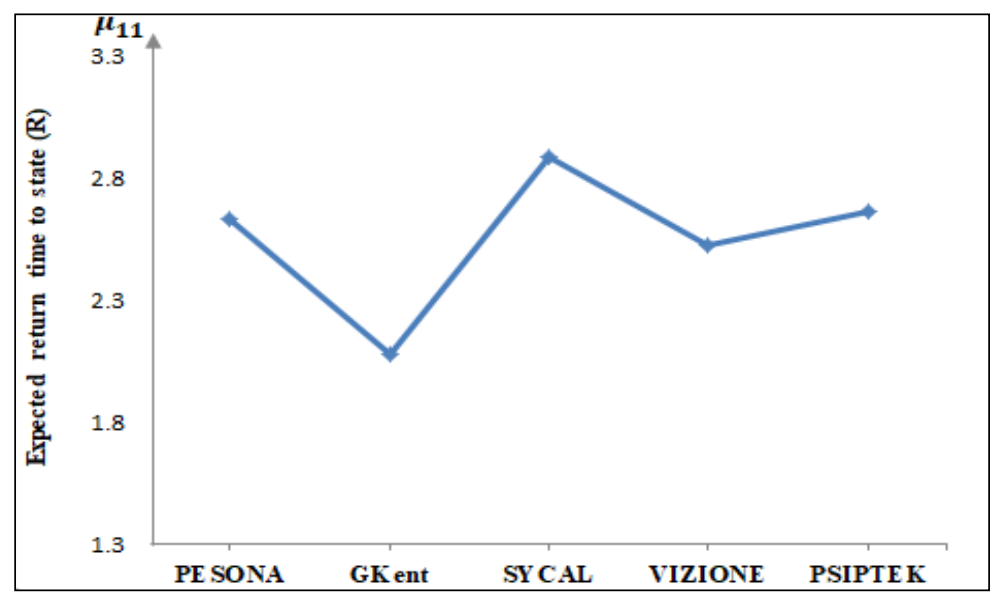

Figure 3. Mean recurrence time of selected shares for three-state

Table 3. Expected return time for respective equities for three-state

\begin{tabular}{|c|ccc|}
\hline Equity & $\boldsymbol{\mu}_{\mathbf{1 1}}=\mathbf{1} / \boldsymbol{\alpha} \mathbf{1}$ & $\boldsymbol{\mu}_{\mathbf{2 2}}=\mathbf{1} / \boldsymbol{\alpha} \mathbf{2}$ & $\boldsymbol{\mu}_{\mathbf{3 3}}=\mathbf{1} / \boldsymbol{\alpha} \mathbf{3}$ \\
\hline PESONA & 2.632895 & 4.952456 & 2.3908 \\
GKent & 2.079997 & 10.1461 & 2.37716 \\
SYCAL & 2.888921 & 4.62214 & 2.285714 \\
VIZIONE & 2.521178 & 4.571429 & 2.600036 \\
PSIPTEK & 2.666667 & 4.952456 & 2.363619 \\
\hline
\end{tabular}

\subsection{Two-State Transition Matrix (Long-Term Behaviour)}

The transition probabilities of a two-state Markov model was also computed and represented in Table 4. These transition probabilities will be used to obtain the limiting distribution of each selected company.

Table 4. Entries of two-state transition matrices for selected equities

\begin{tabular}{|c|cccc|}
\hline Equities & $p_{11}$ & $p_{12}$ & $p_{21}$ & $p_{22}$ \\
\hline PESONA & 0.603306 & 0.396694 & 0.551724 & 0.448276 \\
GKent & 0.576763 & 0.423237 & 0.582857 & 0.417143 \\
SYCAL & 0.529915 & 0.470085 & 0.604396 & 0.395604 \\
VIZIONE & 0.570313 & 0.429688 & 0.68125 & 0.31875 \\
PSIPTEK & 0.508333 & 0.491667 & 0.670455 & 0.329545 \\
\hline
\end{tabular}

The limiting distributions of respective companies were also computed for the two-state Markov chain model in order to identify their long-term behavior. As shown in Table 5, VIZIONE equity has $61 \%$ chance of increasing in the long-term. On the other hand, it has $39 \%$ chance of falling in a long-term. In a similar fashion, the share of GKent has 58\% chance of experiencing increasing, 
International Journal of Mathematical, Engineering and Management Sciences

Vol. 5, No. 2, 296-308, 2020

https://doi.org/10.33889/IJMEMS.2020.5.2.024

but $42 \%$ chance of falling in the long-term. The remaining companies are also interpreted analogously. Based on a two-state model, it can be concluded that VIZIONE company has the highest probability of increasing stock prices in the long-term in comparison to the selected companies in the present study.

Table 5. Limiting distribution at for respective stocks for two-state

\begin{tabular}{|c|cc|}
\hline Equity & $\alpha 1$ & $\alpha 2$ \\
\hline PESONA & 0.5817 & 0.4183 \\
GKent & 0.5793 & 0.4207 \\
SYCAL & 0.5625 & 0.4375 \\
VIZIONE & 0.6136 & 0.3870 \\
PSIPTEK & 0.5769 & 0.4231 \\
\hline
\end{tabular}

The expected return time is also estimated for each selected equity and are indicated in Table 6. As we are seeking to have the least mean return time to a price increase, we plot the mean return time for the selected equities at the state of increasing $\mu_{11}$. From Figure 4 , it appears that VIZIONE equity has the shortest time to return to a price increase. That is, the average time to move to the next increase for VIZIONE is 1.6 weeks.

Table 6. Expected mean return time for respective stocks for two-state

\begin{tabular}{|c|cc|}
\hline Equity & $\mu_{11}=1 / \alpha_{1}$ & $\mu_{22}=1 / \alpha_{2}$ \\
\hline PESONA & 1.719008 & 2.390805 \\
GKent & 1.726141 & 2.377143 \\
SYCAL & 1.777778 & 2.285714 \\
VIZIONE & 1.630734 & 2.585455 \\
PSIPTEK & 1.733333 & 2.363636 \\
\hline
\end{tabular}

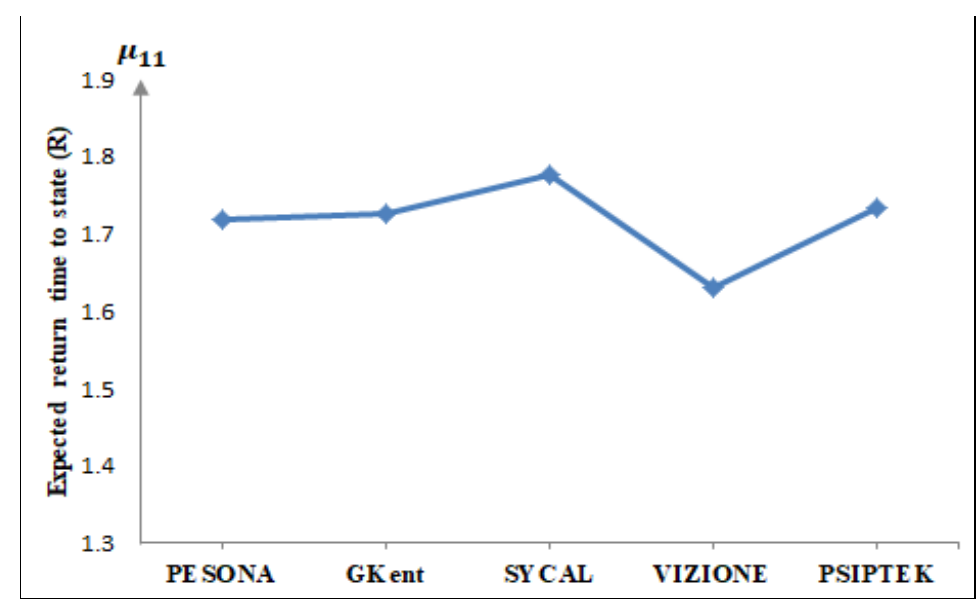

Figure 4. Mean recurrence time of selected shares for two-state 
International Journal of Mathematical, Engineering and Management Sciences

Vol. 5, No. 2, 296-308, 2020

https://doi.org/10.33889/IJMEMS.2020.5.2.024

\section{Conclusion}

The present study forecasts the long-run behavior of some selected public listed companies operating in the Malaysian construction sector. The results reveal that all the companies possess the Markov properties (ergodic and periodic) and have their limiting distributions. The mean return time is also obtained for the respective equities. Investors can make use of the method presented to acquire an appropriate idea about the behavior of the selected shares of respective companies from the transition probabilities, limiting distributions, and the expected mean return time to a price increase. Therefore, the investors are able to choose better shares that will help them achieve higher returns with lower risk.

In the case of a three-state Markov chain model, our results suggest that GKent company has the highest transition probability, the highest limiting distribution, and the shortest mean return time to a price increase (i.e., 2 weeks). In the case of the two-state Markov chain model, however, the results reveal that VIZIONE equity possesses the highest transition probability, the highest limiting distribution, and the lowest mean return time to a price increase.

It can be concluded that studying the long-term behavior of the stock market using the Markov chain models might improve investment decisions, which in turn improve the investor's knowledge and his/her chances of acquiring higher returns, given minimum risk by providing them with better decision choices. Accordingly, our stochastic analysis research tool provides accurate and strong evidence to improve the investment decisions in the Malaysian stock market, especially in the Malaysian construction sector.

\section{Conflict of Interest}

The authors confirm that there is no conflict of interest to declare for this publication.

\section{Acknowledgements}

The authors would like to express their sincere thanks to the editor and anonymous reviews for their time and valuable suggestions. This research was supported in part by the School of Mathematical Sciences at Universiti Sains Malaysia.

\section{References}

Aguilera, A.M., Ocaña, F.A., \& Valderrama, M.J. (1999). Stochastic modelling for evolution of stock prices by means of functional principal component analysis. Applied Stochastic Models in Business and Industry, 15(4), 227-234.

Akbari, A. (2013). The study and evaluation of stocks' valuation models in Tehran stock exchange. European Online Journal of Natural and Social Sciences, 2(3), 2152-2160.

Amiri, A., Ravanpaknodezh, H., \& Jelodari, A. (2016). Comparison of stock valuation models with their intrinsic value in Tehran stock exchange. Marketing and Branding Research, 3, 24-40. SSRN: https://ssrn.com/abstract=3340384.

Bairagi, A., \& Kakaty, S. (October, 2015). Analysis of stock market behaviour: a Markov chain approach. International Journal Recent Scientific Research, 6(10), 7061-7066.

Bhat, U.N. (1984). Elements of applied stochastic processes. Second edition. New York: John Wiley \& Sons. 
International Journal of Mathematical, Engineering and Management Sciences

Vol. 5, No. 2, 296-308, 2020

https://doi.org/10.33889/IJMEMS.2020.5.2.024

Bhusal, M.K. (October, 2017). Application of Markov chain model in the stock market trend analysis of Nepal. International Journal of Scientific \& Engineering Research, 8(10), 1733-1745.

Hassan, M.R., \& Nath, B. (2005, September). Stock market forecasting using hidden Markov model: a new approach. In 5th International Conference on Intelligent Systems Design and Applications (ISDA'05) (pp. 192-196). IEEE. Warsaw, Poland. DOI: 10.1109/ISDA.2005.85

Mettle, F.O., Quaye, E.N.B, \& Laryea, R.A. (2014). A methodology for stochastic analysis of share prices as Markov chains with finite states. SpringerPlus, 3, 657. doi:10.1186/2193-1801-3-657.

Obasi, R., Abdullahi, S.R., \& Ayila, J. (2018). The behaviour of stock market return in two west African capital market: a Markovian analysis. International Journal of Organization \& Business Execellence, $3(1), 21-51$.

Onwukwe, C.E., \& Samson, T.K. (2014). On Predicting the long run behaviour of Nigerian bank stocks prices: a Markov chain approach. American Journal of Applied Mathematics and Statistics, 2(4), 212215.

Sahoo, P.K., \& Charlapally, K. (2015). Stock price prediction using regression analysis. International Journal of Scientific \& Engineering Research, 6(3), 1655-1659.

Xi, X., Mamon, R., \& Davison, M. (2014). A higher-order hidden Markov chain-modulated model for asset allocation. Journal of Mathematical Modelling and Algorithms in Operations Research, 13, 59-85.

Zhang, D., \& Zhang, X. (2009). Study on forecasting the stock market trend based on stochastic analysis method. International Journal of Business Management, 4(6), 163-170. 